\title{
Dixon-based MRI for assessment of muscle-fat content in phantoms, healthy volunteers and patients with achillodynia: comparison to visual assessment of calf muscle quality
}

Fischer, Michael A ; Pfirrmann, Christian W A ; Espinosa, Norman ; Raptis, Dimitri A ; Buck, Florian $\mathrm{M}$

\begin{abstract}
OBJECTIVES: To quantify the muscle fat-content (MFC) in phantoms, volunteers and patients with achillodynia using two-point Dixon-based magnetic resonance imaging (2pt-MRIDIXON) in comparison to MR spectroscopy (MRS) and visual assessment of MFC. METHODS: Two-point Dixonbased MRI was used to measure the MFC of 15 phantoms containing 0-100\% fat-content and calf muscles in 30 patients ( 13 women; $57 \pm 15$ years) with achillodynia and in 20 volunteers (10 women; $30 \pm 14$ years) at $1.5 \mathrm{~T}$. The accuracy of 2 pt-MRIDIXON in quantification of MFC was assessed in vitro using phantoms and in vivo using MRS as the standard of reference. Fat-fractions derived from 2pt-MRIDIXON (FFDIXON) and MRS (FFMRS) were related to visual assessment of MFC (Goutallier grades 0-4) and Achilles-tendon quality (grade 0-4). RESULTS: Excellent linear correlation was demonstrated for FFDIXON with phantoms and with FFMRS in patients $(\mathrm{p} \mathrm{c}=0.997 / 0.995 ; \mathrm{p}<0.001)$. FFDIXON of the gastrocnemius muscle was significantly higher $(\mathrm{p}=0.002)$ in patients $(7.0 \% \pm 4.7$ $\%$ ) compared with volunteers $(3.6 \% \pm 0.7 \%)$, whereas visual-grading showed no difference between both groups $(\mathrm{p}>0.05)$. FFMRS and FFDIXON were significantly higher in subjects with $(>$ grade 1$)$ structural damage of the Achilles-tendon $(\mathrm{p}=0.01)$. CONCLUSIONS: Two-point Dixon-based MRI allows for accurate quantification of MFC, outperforming visual assessment of calf muscle fat. Structural damage of the Achilles tendon is associated with a significantly higher MFC. KEY POINTS: Two-point Dixon-based MRI allows accurate quantification of muscular fat content (MFC). Quantitative analysis outperforms visual analysis in the detection of elevated MFC. Achillodynia results in an increased MFC of the gastrocnemius muscles. Structural damage of the Achilles tendon further increases the MFC.
\end{abstract}

DOI: https://doi.org/10.1007/s00330-014-3121-1

Posted at the Zurich Open Repository and Archive, University of Zurich

ZORA URL: https://doi.org/10.5167/uzh-104555

Journal Article

Published Version

Originally published at:

Fischer, Michael A; Pfirrmann, Christian W A; Espinosa, Norman; Raptis, Dimitri A; Buck, Florian M (2014). Dixon-based MRI for assessment of muscle-fat content in phantoms, healthy volunteers and patients with achillodynia: comparison to visual assessment of calf muscle quality. European Radiology, 24(6):1366-1375.

DOI: https://doi.org/10.1007/s00330-014-3121-1 


\title{
Dixon-based MRI for assessment of muscle-fat content in phantoms, healthy volunteers and patients with achillodynia: comparison to visual assessment of calf muscle quality
}

\author{
Michael. A. Fischer • Christian W. A. Pfirrmann • \\ Norman Espinosa • Dimitri A. Raptis • Florian M. Buck
}

Received: 26 November 2013 / Revised: 24 January 2014 / Accepted: 11 February 2014 / Published online: 7 March 2014

(C) European Society of Radiology 2014

\begin{abstract}
Objectives To quantify the muscle fat-content (MFC) in phantoms, volunteers and patients with achillodynia using twopoint Dixon-based magnetic resonance imaging (2ptMRI $_{\text {DIXON }}$ ) in comparison to MR spectroscopy (MRS) and visual assessment of MFC.

Methods Two-point Dixon-based MRI was used to measure the MFC of 15 phantoms containing 0-100\% fat-content and calf muscles in 30 patients ( 13 women; $57 \pm 15$ years) with achillodynia and in 20 volunteers ( 10 women; $30 \pm 14$ years) at $1.5 \mathrm{~T}$. The accuracy of $2 \mathrm{pt}-\mathrm{MRI}_{\text {DIXON }}$ in quantification of MFC was assessed in vitro using phantoms and in vivo using MRS as the standard of reference. Fat-fractions derived from 2 pt-MRI DIXON $_{\left(F_{\text {DIXON }}\right) \text { and MRS (FF }}$ MRS $)$ were related to visual assessment of MFC (Goutallier grades 0-4) and Achilles-tendon quality (grade 0-4).

Results Excellent linear correlation was demonstrated for $\mathrm{FF}_{\text {DIXON }}$ with phantoms and with $\mathrm{FF}_{\text {MRS }}$ in patients $\left(p_{\mathrm{c}}=\right.$ $0.997 / 0.995 ; p<0.001) . \mathrm{FF}_{\mathrm{DIXON}}$ of the gastrocnemius muscle was significantly higher $(p=0.002)$ in patients $(7.0 \% \pm 4.7 \%)$ compared with volunteers $(3.6 \% \pm 0.7 \%)$, whereas visualgrading showed no difference between both groups $(p>0.05)$. $\mathrm{FF}_{\mathrm{MRS}}$ and $\mathrm{FF}_{\text {DIXON }}$ were significantly higher in subjects with (>grade 1) structural damage of the Achilles-tendon ( $p=$ $0.01)$.
\end{abstract}

M. A. Fischer $(\bowtie) \cdot$ C. W. A. Pfirrmann • F. M. Buck

Radiology, University Hospital Balgrist, Forchstrasse 340, 8008 Zurich, Switzerland

e-mail: michaelalexander.fischer@usz.ch

\section{N. Espinosa}

Department of Orthopedic Surgery, University Hospital Balgrist,

Forchstrasse 340, 8008 Zurich, Switzerland

D. A. Raptis

Clinic of Visceral and Transplant Surgery, University Hospital

Zurich, Raemistrasse 100, 8091 Zurich, Switzerland
Conclusions Two-point Dixon-based MRI allows for accurate quantification of MFC, outperforming visual assessment of calf muscle fat. Structural damage of the Achilles tendon is associated with a significantly higher MFC.

Key points

- Two-point Dixon-based MRI allows accurate quantification of muscular fat content (MFC).

- Quantitative analysis outperforms visual analysis in the detection of elevated MFC.

- Achillodynia results in an increased MFC of the gastrocnemius muscles.

- Structural damage of the Achilles tendon further increases the MFC.

Keywords Achilles tendon · Muscle · Fat quantification · Two-point Dixon technique $\cdot$ Magnetic resonance imaging

\begin{tabular}{|c|c|}
\hline Abbreviations & \\
\hline MFC & Muscle fat content \\
\hline $2 p t-M_{\text {DIXON }}$ & Two-point Dixon-based MRI \\
\hline FF & Fat fraction \\
\hline
\end{tabular}

\section{Introduction}

Magnetic resonance imaging (MRI) is considered a reliable technique for evaluating muscle quality by monitoring morphological changes like muscular atrophy and fatty degeneration [1]. MR spectroscopy (MRS), which provides the reference standard of non-invasive quantification of fat, has been challenged by two-point Dixon-based MRI (2pt-MRI DIXON $_{\text {) }}$ with chemical shift selective reconstruction of fat- and watersignal only image series [2]. Two-point Dixon-based MRI promises significant advantages over MRS including fast volumetric coverage of muscular anatomy with the possibility 
of postprocessing capabilities such as multiplanar reformations [3]. Moreover, muscular fat content (MFC) can be assessed retrospectively by determining a region of interest (ROI) to eliminate sampling errors, which could result from inadequate positioning of the MRS voxel or inadequate site of biopsy [4].

Achillodynia is the most common pain-related disability in sport and either caused by an injury of the Achilles tendon itself or by an inflammatory process occurring in the surrounding tissues [5]. Concerning pathophysiology, a series of mechanisms reducing tissue perfusion and mechanical irritation lead to degeneration of the tendon [6]. Fatty degeneration of the calf muscles is a common finding in patients with tendinosis [7] and is probably the result of altered musculoskeletal function. Vice versa, abnormal muscle function is speculated to be a risk factor for Achilles tendinopathy [8]. Similar to the rotator cuff in the shoulder, the quality of the soleus and gastrocnemius muscles may be a predictive factor for the outcome after treatment of the Achilles tendon [9, 10]. Accurate quantification of the calf muscle fat content in patients with achillodynia could therefore aid assessment of muscular function prior to surgery or prior to rehabilitation programs and serve as a biomarker for disease progress.

Thus, the objectives of this prospective study were: (1) to evaluate the accuracy of a standard 2 pt-MRI $I_{\text {DIXON }}$ product sequence for quantification of MFC and (2) to quantify the MFC of the gastrocnemius and the tibialis anterior muscle in patients with achillodynia and healthy volunteers using 2ptMRI $_{\text {DIXON }}$ and MRS in comparison to visual assessment of calf muscle quality.

\section{Material and methods}

Phantom study

\section{Phantoms}

The in vitro study included 15 polypropylene test tubes (Falcon; Becton Dickinson, Franklin Lakes, NJ, USA) containing $10 \mathrm{ml}$ of homogenised mixtures of muscle tissue (veal meat) and fat (goose fat) at concentrations of 0, 2, 4, 6, 8, 10, 20, 30, $40,50,60,70,80,90$ and $100 \%$ as well as one control test tube $(\mathrm{C})$ containing pure and untreated muscle tissue. All test tubes were placed in a plastic box $(22 \times 30 \times 40 \mathrm{~cm})$ filled with a solution of water and gadolinium-based contrast medium $(0.25 \%)$ (Fig. 1).

Sample preparation was performed according to a previous study evaluating liver fat [11]: muscle tissue was cleaned from extracellular fat and blood remnants using a heparin/water (1/10) solution (heparin, 25,000 IU/5 ml), homogenised mechanically in a commercially available mixer (Braun PowerBlend MX 2000; Braun, Pfungstadt, Germany) and quantitatively titrated (volume \%) using a 5-ml multisampler (Multipett plus; Eppendorf, Hamburg, Germany). Liquid goose fat was titrated up to $10 \mathrm{ml}$ using a $1-\mathrm{ml}$ pipette and both components were homogenised and centrifuged thereafter.

Veal meat was chosen as a target matrix since it closely matches human muscle tissue and does not contain significant intracellular fat [12], whereas goose fat was chosen due to its low melting point (approx. $35^{\circ} \mathrm{C}$ ) and high percentage of organic fat (approx. $99 \%$ ) allowing for good sample homogenisation and accurate fat titration.

\section{Data acquisition}

Two-point Dixon gradient-echo MRI with an automatic chemical-shift selective reconstruction algorithm was performed on a 1.5-T MRI system (Avanto; Siemens Medical Healthcare, Erlangen, Germany) using a dedicated body-array coil. This algorithm was shown to provide stable water- and fat-signal separation for dual-echo imaging using phase information to resolve the ambiguity of fat and water signal in chemical-shift imaging [3, 13]. Sequence specifications and imaging parameters are given in Table 1.

\section{Data analysis}

Data analysis was performed using Osirix (version 5.5.1; OsiriX Imaging Software, open-source DICOM viewer, www.osirix-viewer.com). Fat- and water-image sets (Fig. 1) were loaded and automatically co-registered in the viewer function of OsiriX. One musculoskeletal radiologist (M.A.F., 5 years of experience), who was blinded to the known MFC of the phantoms, evaluated both image sets by placing an ROI along the coronal plane of each phantom. ROIs were copied to the corresponding image set and carefully adjusted to cover the entire volume of the phantom without integrating external structures.

For each phantom, the fat fraction $\left(\mathrm{FF}_{\mathrm{DIXON}}\right)$ was calculated from signal intensities derived from fat-only images $\left(\mathrm{SI}_{\mathrm{FAT}}\right)$ and water-only images ( $\mathrm{SI}_{\text {WATER }}$ ) according to the following algorithm:

$\mathrm{FF}_{\mathrm{DIXON}}=\mathrm{SI}_{\mathrm{FAT}}\left(\mathrm{SI}_{\mathrm{FAT}}+\mathrm{SI}_{\mathrm{WATER}}\right)$

In vivo study

This was a prospective study with local ethics committee approval. Written informed consent was obtained from all study subjects. 
Fig. 1 In vitro measurement of Dixon-based MRI fat fractions. a Muscle/fat phantoms and corresponding MR image sets derived from a 2-point Dixon gradient-echo MRI sequence with automatic reconstruction of (b) water- and (c) fat-only images in the axial plane (muscle fat content: $0,2,4,6,8,10,20,30$, $40,50,60,70,80,90$ and $100 \%$; $C$, control)

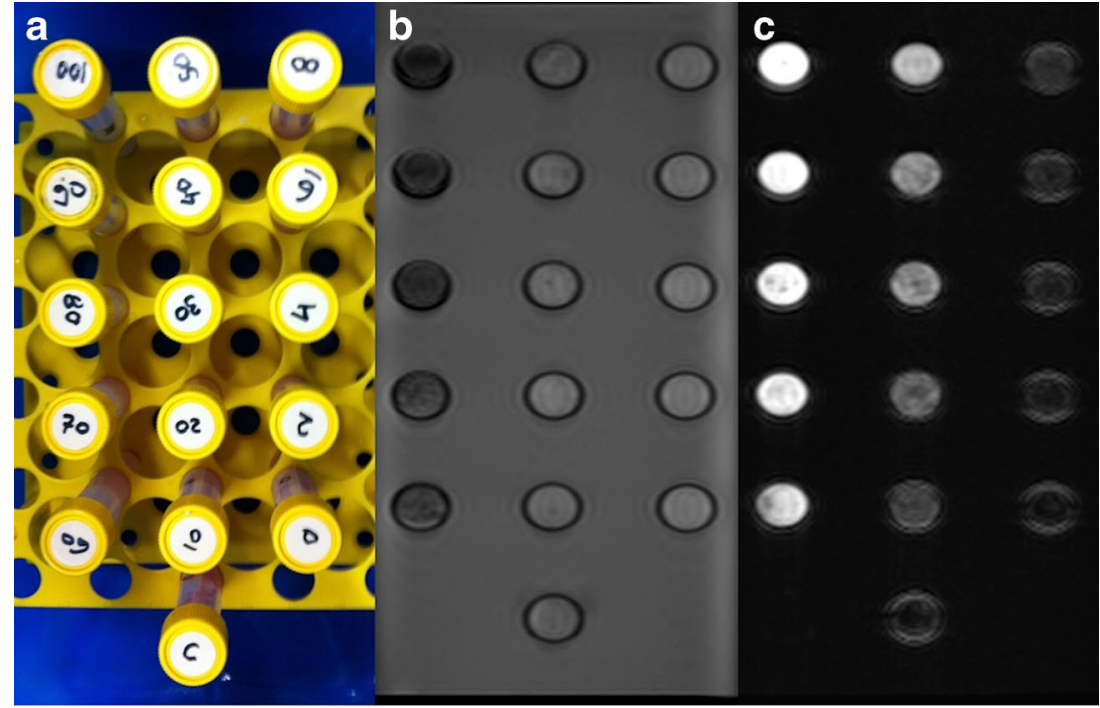

\section{Patients}

The patient group consisted of 30 consecutive patients (12 women, 18 men; mean age, $57 \pm 15$ years; age range, 2786 years) who underwent MRI of the Achilles tendon due to achillodynia. Inclusion criteria were a clinical diagnosis of achillodynia by the referring clinician, which was defined as Achilles tendon pain of more than 2 months in duration. Exclusion criteria were general contraindication for MRI, prior surgery, neurological disorders, systemic inflammatory disease, acute trauma and disorders of the ankle other than Achilles tendon disease.

\section{Healthy volunteers}

The control group consisted of 20 asymptomatic volunteers ( 9 women, 11 men; mean age, $49 \pm 14$ years; age range, 30-76 years) matched to the patient group according to sex. Inclusion criteria were absence of achillodynia within the past 2 years. Professional and semi-professional athletes were excluded from the control group and volunteers were asked to rest for at least $24 \mathrm{~h}$ before examination, as exercise might potentially bias Dixon-based measurements [14]. All other exclusion criteria equalled the patient group.

Table 1 MRI protocol and corresponding imaging parameters

\begin{tabular}{|c|c|c|c|c|c|c|}
\hline \multirow[t]{2}{*}{ Imaging technique } & \multicolumn{3}{|c|}{ Achilles tendon } & \multicolumn{3}{|c|}{ Calf muscles } \\
\hline & T1w-SE & T2w-TSE & STIR & T1w-TSE & STIR & 2pt-DixonGRE \\
\hline Orientation & Sagittal & Sagittal & Axial & Axial & Axial & Axial \\
\hline No. of dimensions & Two & Two & Two & Two & Two & Three \\
\hline Repetition time (ms) & $439-473$ & $4,400-4,640$ & 4,000 & 610 & 5,420 & 16.2 \\
\hline Echo time $^{\mathrm{a}}(\mathrm{ms})$ & 13 & 77 & 78 & 11 & 78 & $2.4 ; 4.8$ \\
\hline No. of signals acquired & 2 & 9 & 7 & 3 & 7 & 2 \\
\hline Flip angle (degrees) & 150 & 180 & 180 & 150 & 180 & 5 \\
\hline Number of averages & 1 & 1 & 2 & 2 & 2 & 1 \\
\hline Section thickness (mm) & 3 & 3 & 6 & 10 & 10 & 5 \\
\hline Spacing & 0.3 & 0.3 & 1.8 & 3 & 3 & 0 \\
\hline Matrix size & $512 \times 358$ & $512 \times 256$ & $256 \times 218$ & $512 \times 265$ & $256 \times 218$ & $256 \times 208$ \\
\hline Bandwidth (Hz/pixel) & \pm 150 & \pm 65 & \pm 130 & \pm 150 & \pm 130 & \pm 456 \\
\hline Number of images & $23-25$ & $23-25$ & $22-24$ & $26-30$ & $26-30$ & $55-64$ \\
\hline
\end{tabular}

2pt-Dixon two-point Dixon-based reconstruction of fat- and water-only images, 3pt-Dixon three-point Dixon-based reconstruction of fat- and water-only images, GRE gradient-echo sequence, SE spin-echo sequence, STIR short TI inversion recovery sequence, TSE turbo spin-echo sequence, TIw T1weighted imaging, $T 2 w$ T2-weighted imaging

${ }^{\text {a }}$ Effective echo time for all SE-based sequences 


\section{Data acquisition}

All study subjects underwent standard clinical MRI of the Achilles tendon and additional Dixon-based MRI as well as MRS using the same 1.5-T MR system (Avanto; Siemens Medical Solutions, Erlangen, Germany) as in the phantom study (Fig. 2). Patients and volunteers were examined in the supine position with a dedicated transmit-receive extremity coil for the Achilles tendon and phased-array body coil (both from Siemens Medical Solutions, Erlangen, Germany) for imaging the calf muscles.

The study protocol consisted of the standard clinical MRI protocol at our department for the examination of patients with achillodynia as well as the previously mentioned 2pt$\mathrm{MRI}_{\text {Dixon }}$ sequence. Imaging parameters are displayed in Table 1. In addition we performed a high-speed T2-corrected multi-echo ${ }^{1} \mathrm{H}$ MRS, serving as the standard of reference [15]. Spectra were obtained in two different locations by placing an $8-\mathrm{cm}^{3}$ voxel $(20 \times 20 \times 20 \mathrm{~mm})$ in the medial or lateral head of the gastrocnemius muscle and in the centre of the tibialis anterior muscle. Voxel placement was performed on transversal standard clinical T1-weighted MR images avoiding muscle fascia and vessels and position of the voxel was saved as DICOM file along with the corresponding spectral analysis. The MRS sequence applied is based on a single-voxel stimulated-echo acquisition mode (STEAM) using the following parameters: repetition time, 3,000 ms; echo times, 12/24/ 36/48/72 ms; acquired points, 1,024; bandwidth, 1,200 Hz; mixing time, $10 \mathrm{~ms}$. The algorithm integrated the water (from 3.6 to $5.8 \mathrm{ppm}$ ) and lipid (methylene and methyl signals of $\alpha$ $\mathrm{CH}_{2},\left(\mathrm{CH}_{2}\right)_{\mathrm{n}-2}$, and $\mathrm{CH}_{3}$ from 0 to $3.6 \mathrm{ppm}$ ) signals for each TE and spectral integrals $(S)$ were measured at each TE. Monoexponential curve fits were automatically performed with least-squares approximation by using the equation $S=$ M0 $\exp (-\mathrm{R} 2 \times \mathrm{TE})$ to estimate R2 and M0 (the equilibrium magnetisation) for water and lipid signals individually [15].

\section{Data analysis}

Quantitative analysis The quantitative analysis was performed by the same radiologist (M.A.F.) doing the ex vivo measurement, blinded to the results of the qualitative image analysis.

For image-based assessment of the MFC, the fat- and water-image sets derived from 2pt-Dixon of each patient were loaded simultaneously with the reference images of spectroscopic ROI positioning into the viewer function of OsiriX (Fig. 2). An ROI with in-plane dimensions matching those of the MRS voxel was placed in the reference image (overlaying the MRS voxel) and copied to the corresponding fat-
Fig. 2 In vivo measurement of fat-fractions derived from 2-point Dixon gradient-echo MRI

$\left(\mathrm{FF}_{\mathrm{DIXON}}\right)$ and MR-spectroscopy $\left(\mathrm{FF}_{\mathrm{MRS}}\right)$ in a 77 -year-old male patient with achillodynia.

FF $_{\text {DIXON }}(15 \%)$ were calculated by placing an ROI in the gastrocnemius muscle on both the fat- (a) and water- (b) only image corresponding to the spectroscopic voxel (d). $\mathrm{FF}_{\mathrm{MRS}}$ $(16 \%)$ integrated the water (from 3.6 to $5.8 \mathrm{ppm}$ ) and lipid (methylene and methyl signals of $\alpha-\mathrm{CH}_{2},\left(\mathrm{CH}_{2}\right)_{\mathrm{n}-2}$, and $\mathrm{CH}_{3}$ from 0 to $3.6 \mathrm{ppm}$ ) signals of the spectral analysis (d). In comparison, T1weighted images (c) were evaluated semi-quantitatively for fatty infiltration according to Goutallier (grade 2)
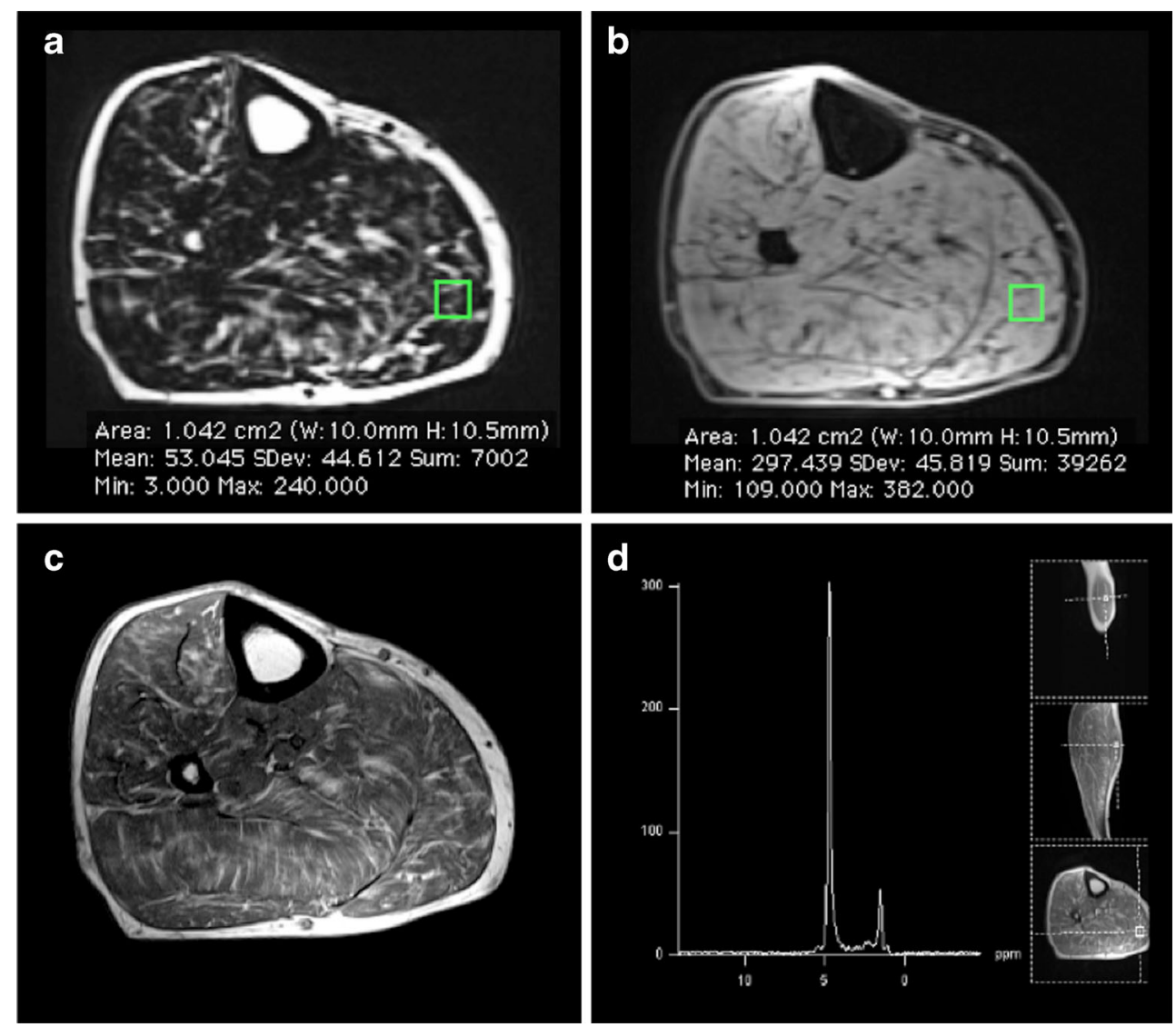
and water-signal only images. Fat fractions $\left(\mathrm{FF}_{\mathrm{DIXON}}\right)$ were then calculated separately for both muscles according to the previously mentioned algorithm (Eq. 1).

Spectroscopic assessment of MFC was performed automatically by an integrated post-processing algorithm yielding $\mathrm{T} 2$ corrected spectroscopic fat fractions $\left(\mathrm{FF}_{\mathrm{MRS}}\right)$ of both muscles as well as R2 of water- and fat- signal. $\mathrm{FF}_{\mathrm{MRS}}$ was calculated equally to the Dixon-based algorithm (Eq. 1).

Qualitative analysis Two independent musculoskeletal radiologists (F.M.B. with 9 years of experience and C.W.P. with 14 years of experience), who were blinded to all other findings but the clinical MRI protocol, performed the qualitative analysis. Both readers visually assessed the quality of the Achilles tendon as well as the quality of the gastrocnemius and the tibialis anterior muscle in two reading sessions with a gap of 6 weeks between both sessions. All images were interpreted on a standard workstation using an integrated picture archiving and communication system (IMPAX 6; Agfa HealthCare, Bonn, Germany) and were presented to the readers with the minimum of annotations allowed by the software.

The quality of the Achilles tendon was rated on a five-point scale [5] with the following specifications: 0 , "normal tendon", concave shape and no T1-/T2- signal alterations; 1 , "paratendinosis", hyperintense signal of the paratendinous soft tissue on STIR-images; 2, "tendinosis", tendon thickening, convex spindle-shape, normal signal or hyperintense areas on T1-weighted images; 3, "partial tear", tendon thickening, hyperintense on $\mathrm{T} 1-$ and $\mathrm{T} 2-$ weighted images; 4, "complete tear", discontinuity of the Achilles tendon.

Extent of fatty degeneration of the gastrocnemius muscle and the tibialis anterior muscle was estimated on T1-weighted images using the five-point scale according to Goutallier et al. [16]: 0 , none; 1 , subtle fatty degeneration; 2 , muscle tissue > fatty degeneration; 3 , muscle tissue $\approx$ fatty degeneration; 4 , fatty degeneration $>$ muscle tissue.

\section{Statistical analysis}

The data was descriptively reviewed and statistically tested for normality with the Kolmogorov-Smirnov test. All results were expressed as means \pm standard deviations; $p$ values $<0.05$ were considered statistically significant. All statistical analyses were performed using commercially available software (release 20.0; SPSS, Chicago, IL USA).

\section{Quantitative analysis (continues variables, normal distribution)}

Correlations between $\mathrm{FF}_{\mathrm{DIXON}}$ and titrated MFC of the phantom study as well as $\mathrm{FF}_{\mathrm{MRS}}$ of the in-vivo study were assessed using Lin's concordance correlation coefficient $\left(p_{\mathrm{c}}\right)$ with $95 \%$ confidence interval (CI) [17], whereas correlation between $\mathrm{FF}_{\text {DIXON }}$ and study subjects' gender and age was assessed with the Spearman's rank correlation coefficient $(\rho)$. Linear regression analysis was used to evaluate the goodness of fit of the linear regression model.

Student's $t$-test was used to test for significant differences between patients and volunteers as well as between different groups of tendinosis.

\section{Qualitative analysis (ordinal variables)}

Inter-observer agreement was assessed by using kappastatistics $(\kappa)$ and interpreted as follows: a $\mathrm{K}$ value greater than 0.81 corresponded to excellent agreement, a $k$ value of 0.61 0.80 corresponded to good interobserver agreement, a $\mathrm{K}$ value of 0.41-0.60 corresponded to moderate interobserver agreement, and a $\mathrm{K}$ value of $0.21-0.40$ corresponded to poor interobserver agreement. Data of the consensus readings was used for further analysis.

The Mann-Whitney $U$ test was used to evaluate significant differences in visual grading of fatty muscle infiltration between patients and healthy volunteers.

\section{Results}

Quantitative analysis

\section{Phantom study}

$\mathrm{FF}_{\text {DIXON }}$ showed excellent correlation with titrated MFC from 0 to $100 \%\left(p_{\mathrm{c}}, 0.997 ; 95 \% \mathrm{CI}, 0.993-0.999 ; p<0.001\right)$. Linear regression analysis revealed a slope of 0.95 and an intercept with the $y$-axis of 1.84 , with a corresponding standard error of the estimated parameters of 0.02 and 0.80 respectively (both, $p<0.05$ ).

$\mathrm{FF}_{\text {DIXON }}$ showed a good correlation with titrated MFC in a clinically relevant range from 0 to $30 \%$ $\left(p_{\mathrm{c}}, 0.971 ; 95 \% \mathrm{CI}, 0.918-0.990 ; p<0.001\right)$. Linear regression analysis revealed a slope of 0.84 and an intercept with the $y$-axis of 2.73 , with a corresponding standard error of the estimated parameters of 0.04 and 0.56 respectively (both, $p<0.01$ ) (Fig. 3).

In vivo study

$\mathrm{FF}_{\mathrm{DIXON}}$ and $\mathrm{FF}_{\mathrm{MRS}}$ were obtained in 30 patients and 20 volunteers within the gastrocnemius muscle and tibialis anterior muscle. Results of quantitative MFC analysis using MRS and 2pt-Dixon ${ }_{\text {GRE }}$ are given in Table 2.

Good correlation was observed between $\mathrm{FF}_{\text {DIXON }}$ and $\mathrm{FF}_{\text {MRS }}\left(p_{\mathrm{c}}, 0.951 ; 95 \% \mathrm{CI}, 0.927-0.973 ; p<0.001\right)$. There was no significant difference between $\mathrm{FF}_{\mathrm{DIXON}}$ and $\mathrm{FF}_{\mathrm{MRS}}$ 


\section{a}

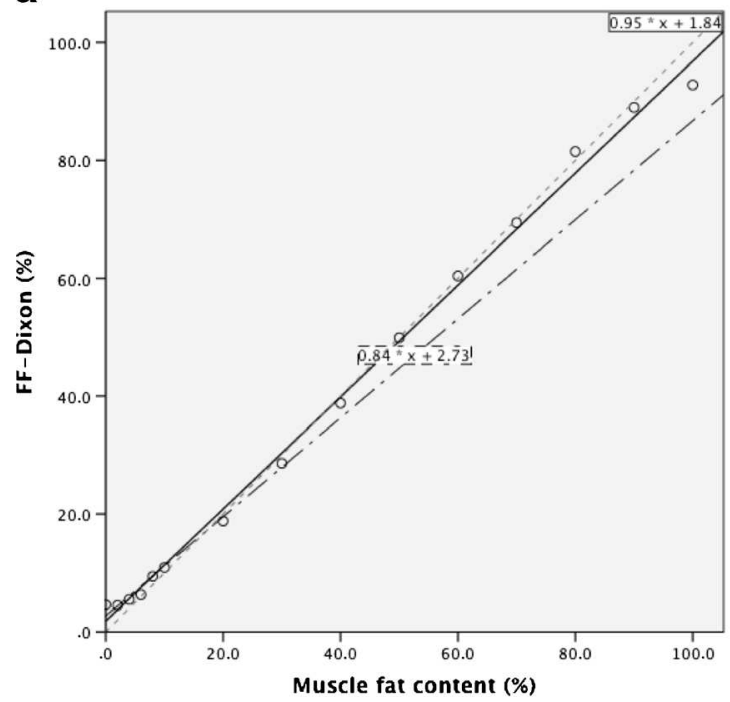

Fig. 3 Correlation of fat-fractions derived from 2-point Dixon gradientecho MRI $\left(\mathrm{FF}_{\mathrm{DIXON}}\right)$ with known muscle fat content (MFC) of the phantoms (a) and MFC of the in vivo study (b) derived from MRS $\left(\mathrm{FF}_{\mathrm{MRS}}\right)$. Data are displayed as scatterplots with linear regression lines (bold line) and corresponding reference lines (dotted line). $\mathrm{FF}_{\mathrm{DIXON}}$ showed excellent and good correlation with titrated MFC of the ex vivo study from 0 to $100 \%\left(p_{c}, 0.997 ; 95 \% \mathrm{CI}, 0.993-0.999\right)$ and from 0 to

$(p>0.05)$ with a mean measurement bias of $+0.4 \%$ (limits of agreement, $-2.8 \%$ to $+3.7 \%$ ). Linear regression analysis revealed a slope of 0.85 and an intercept with the $y$-axis of 0.68 , with a corresponding standard error of 0.04 and 0.23 (both, $p<0.01$ ) (Fig. 3).

Student's $t$-test revealed a significantly higher $\mathrm{FF}_{\mathrm{DIXON}}$ of the gastrocnemius muscle in patients compared with volunteers $(p=0.002)$, whereas no difference was identified for $\mathrm{FF}_{\mathrm{DIXON}}$ of the tibialis anterior muscle between both groups $(p>0.05)$ (Fig. 4). There was no significant correlation between $\mathrm{FF}_{\mathrm{DIXON}}$ of the gastrocnemius and tibialis anterior muscles, visual-grading of the Achilles tendon quality and characteristics of study subjects (gender and age) for all constellations $(\rho, 0.05-0.32 ; p>0.05)$, except $\mathrm{FF}_{\mathrm{DIXON}}$ and age of patients and volunteers $(p=0.02 ; p=0.006)$. However, the latter showed poor correlation coefficients $(\rho)$ for both of 0.49 and 0.50 respectively. b

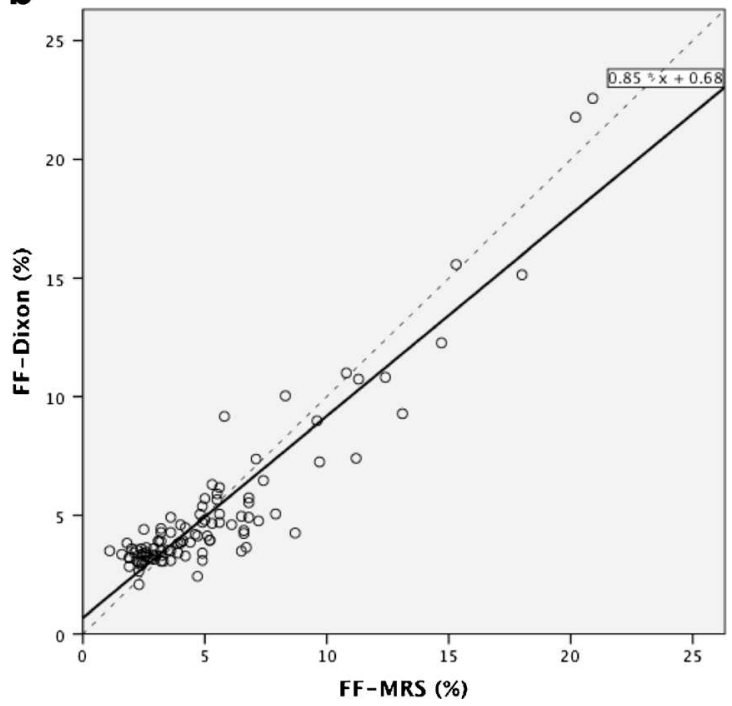

$30 \% \operatorname{MFC}\left(p_{\mathrm{c}}, 0.971 ; 95 \% \mathrm{CI}, 0.918-0.990 ; p<0.001\right)$ respectively, as well as with the $\mathrm{FF}_{\mathrm{MRS}}\left(p_{\mathrm{c}}, 0.951 ; 95 \% \mathrm{CI}, 0.927-0.973 ; p<0.001\right)$ of the in vivo study. Linear regression analysis revealed a slope of (a) 0.95 (black line; MFC 0-100\%) and 0.84 (black semi-dotted line; MFC 0$30 \%$ ) in the ex vivo study and (b) 0.85 (black line; MFC 1.1-22.6\%) in the in vivo study respectively

Qualitative analysis

Inter-observer agreement was excellent for visual assessment of Achilles tendon quality $(\kappa=0.811 ; p<0.001)$; good for visual grading of the fatty degeneration of the gastrocnemius muscle $(\kappa=0.633 ; p<0.001)$; and moderate for visual grading of the tibialis anterior muscle $(\mathrm{K}=0.542 ; p<0.001)$.

\section{Achilles tendon quality}

A "normal" Achilles tendon (grade 0) was present in 5/30 (17\%) of patients and 16/20 (80\%) of volunteers. Paratendinosis (grade 1) was identified in 4/30 (13\%) of patients and 4/20 (20\%) of volunteers. Tendinosis (grade 2), partial tear (grade 3 ) and complete tear (grade 4) were identified in $10 / 30(33 \%), 7 / 30(23 \%)$ and $4 / 30(13 \%)$ of patients, respectively, but in none of the volunteers.

Table 2 Mean fat fractions as derived from MRS $\left(\mathrm{FF}_{\mathrm{MRS}}\right)$ and Dixon-based MRI ( $\left.\mathrm{FF}_{\mathrm{DIXON}}\right)$ in comparison with mean values of visual grading and transverse relaxation rate (R2) of fat derived from MRS

\begin{tabular}{|c|c|c|c|c|c|c|}
\hline & \multicolumn{3}{|c|}{ Gastrocnemius muscle } & \multicolumn{3}{|c|}{ Tibialis anterior muscle } \\
\hline & Patients & Volunteers & $p$ value & Patients & Volunteers & $p$ value \\
\hline $\mathrm{FF}_{\mathrm{MRS}}$ & $7.9 \pm 5.2$ & $4.9 \pm 2.9$ & 0.002 & $3.9 \pm 1.2$ & $3.1 \pm 1.3$ & $>0.05$ \\
\hline $\mathrm{FF}_{\text {DIXON }}$ & $7.0 \pm 4.7$ & $5.0 \pm 2.3$ & 0.002 & $3.6 \pm 0.7$ & $3.6 \pm 0.5$ & $>0.05$ \\
\hline Visual grade $^{a}$ & $0.9 \pm 0.8$ & $0.6 \pm 0.5$ & $>0.05$ & $0.6 \pm 0.9$ & $0.3 \pm 0.5$ & $>0.05$ \\
\hline R2 (fat) & $27.3 \pm 4.3$ & $31.7 \pm 2.7$ & $<0.001$ & $32.3 \pm 3.0$ & $34.5 \pm 1.3$ & 0.014 \\
\hline
\end{tabular}

Mean values are displayed as averages with corresponding standard deviations

${ }^{\mathrm{a}}$ Visual grades ranged from 0-3 according to Goutallier et al. [16] 
Fig. 4 Muscle fat content (MFC) as determined by 2-point Dixon gradient-echo MRI ( $\mathrm{FF}_{\text {DIXON }}$ ) and MRS ( $\left.\mathrm{FF}_{\mathrm{MRS}}\right) . \mathrm{FF}_{\mathrm{DIXON}}$ and $\mathrm{FF}_{\mathrm{MRS}}$ of the gastrocnemius muscle were significantly higher in (0) patients compared with (1) volunteers $(p=0.002 / p=0.001)$, whereas no difference was identified for $\mathrm{FF}_{\text {DIXON }}$ and $\mathrm{FF}_{\mathrm{MRS}}$ of the tibialis anterior muscle between both groups (both, $p>0.05$ ). Data are displayed as box-and-whisker plots. Boundary of boxes closest to zero 25th percentile, line within box median, boundary of boxes farthest from zero 75 th percentile; error bars smallest and largest values within 1.5 box lengths of 25th and 75 th percentiles

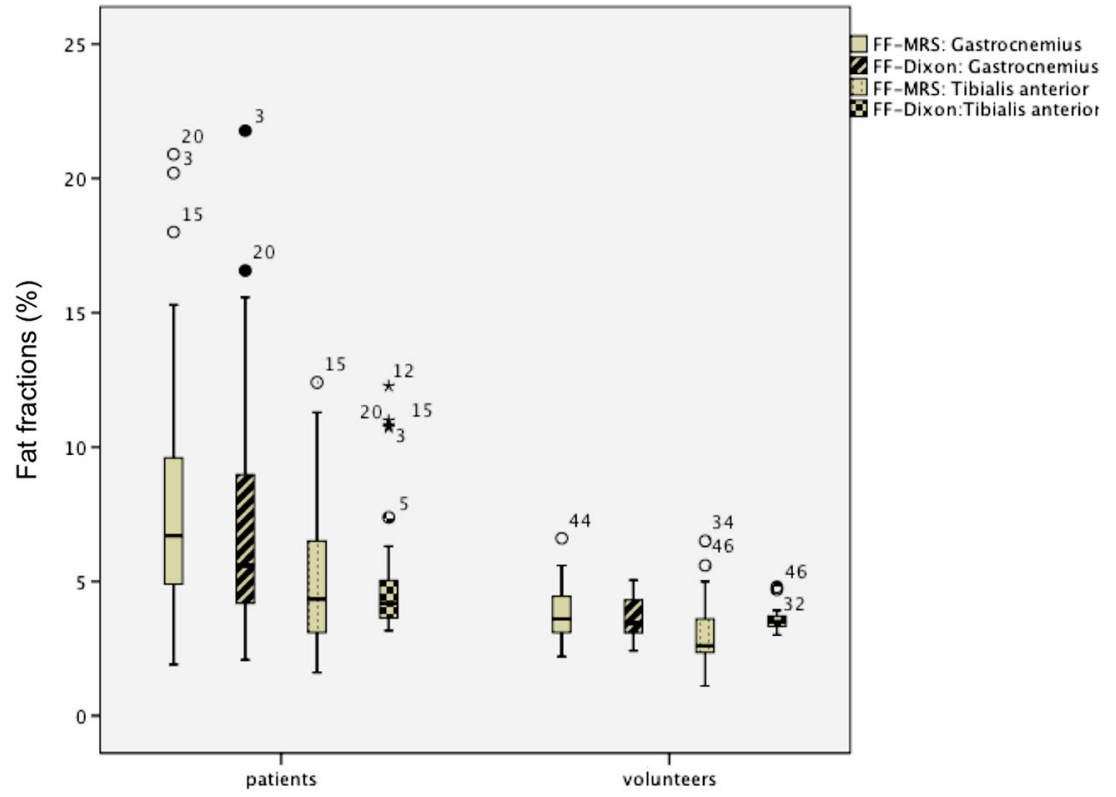

Distribution of MFC in respect to Achilles tendon quality is displayed in Fig. 5. Student's $t$-test revealed a significantly higher $\mathrm{FF}_{\mathrm{MRS}}$ and $\mathrm{FF}_{\mathrm{DIXON}}$ in study subjects with (> grade 1) than without $(\leq$ grade 1$)$ structural damage of the Achilles tendon ( $p=0.01)$, whereas no significant difference was seen between study subjects with paratendinosis (grade 1) and normal (grade 0 ) Achilles tendon $(p>0.05)$.

\section{Fatty muscle degeneration}

Subtle fatty degeneration (Goutallier grade 1) of the gastrocnemius/tibialis anterior muscle was detected in $15 / 30$

a

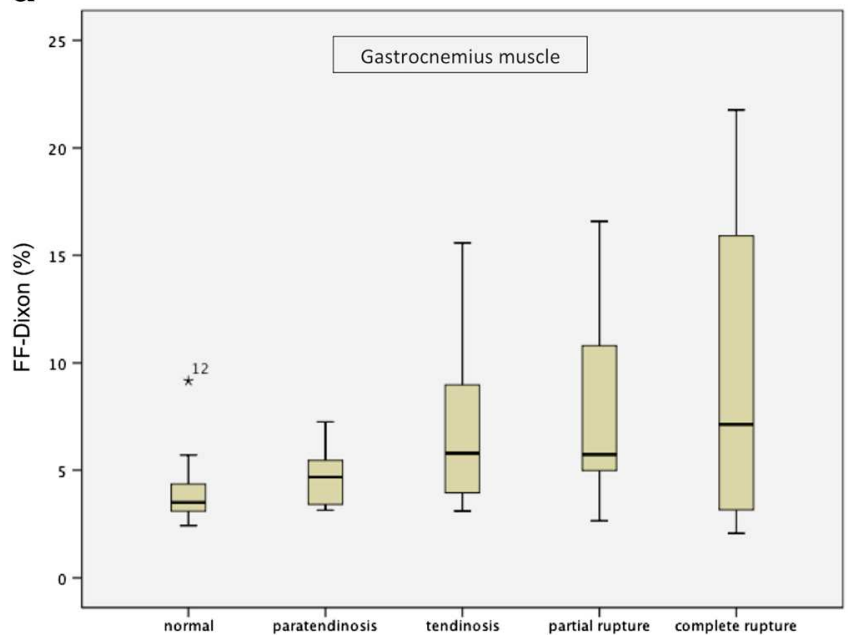

Fig. 5 Distribution of muscle fat content as determined by 2-point Dixon gradient-echo MRI ( $\left.\mathrm{FF}_{\mathrm{DIXON}}\right)$ along the $y$-axis in respect to Achilles tendon quality (grade $0-4$ ) along the $x$-axis. $\mathrm{FF}_{\mathrm{DIXON}}$ of the gastrocnemius muscle (a) was significantly higher in study subjects with ( $>$ grade 1) compared to without ( $\leq$ grade 1$)$ structural damage of the Achilles tendon $(p=0.01)$, whereas no significant differences were seen for $\mathrm{FF}_{\mathrm{DIXON}}$ of
$(50 \%) / 10 / 30(33 \%)$ of patients and 12/20 (60\%) / 6/20 (30\%) of volunteers respectively. Goutallier grade 2 was identified in 5/30 (17\%)/ 3/30 (10\%) of patients, whereas Goutallier grade 3 was identified in $1 / 30(3 \%)$ and $0 / 30(0 \%)$ patients. None of the volunteers showed $>$ grade 1 and none of the patients showed grade 4 fatty degeneration of muscle tissue. In total 9/30 (30\%)/ 17/30 (57\%) of patients and $8 / 20(40 \%) / 14 / 20(70 \%)$ of volunteers showed no signs of fatty degeneration. The Mann-Whitney $U$ test showed no significant differences of visual grading between patients and volunteers $(p>0.05) . \mathrm{FF}_{\mathrm{MRS}}$ and $\mathrm{FF}_{\mathrm{DIXON}}$ were significantly different between Goutallier grades 0,1 and 2 respectively (all,

b

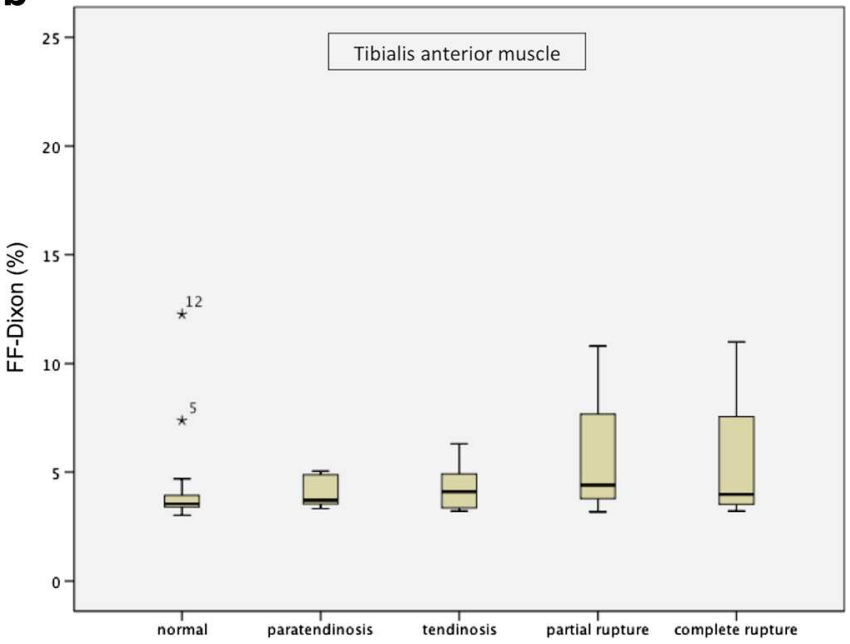

the tibialis anterior muscle (b) between same groups $(p>0.05)$. Data are displayed as box-and-whisker plots. Boundary of boxes closest to zero 25th percentile, line within box median, boundary of boxes farthest from zero 75 th percentile; error bars smallest and largest values within 1.5 box lengths of 25 th and 75 th percentiles 
Table 3 Mean fat fractions as derived from MRS $\left(\mathbf{F F}_{\mathbf{M R S}}\right)$ and Dixon-based MRI $\left(\mathbf{F F}_{\mathbf{D I x O N}}\right)$ in regards to Goutallier grades derived from visual grading of muscle fat content

\begin{tabular}{|c|c|c|c|c|}
\hline \multirow[t]{2}{*}{ Goutallier grade } & \multicolumn{2}{|c|}{ Gastrocnemius muscle } & \multicolumn{2}{|c|}{ Tibialis anterior muscle } \\
\hline & $\mathrm{FF}_{\mathrm{MRS}}$ & $\mathrm{FF}_{\text {DIXON }}$ & $\mathrm{FF}_{\mathrm{MRS}}$ & $\mathrm{FF}_{\mathrm{DIXON}}$ \\
\hline 0 & $3.6(1.9-6.8)$ & $3.5(2.1-5.7)$ & $3.6(1.1-8.7)$ & $4.0(3.0-7.4)$ \\
\hline 1 & $5.9(2.4-13.1)$ & $5.0(2.4-9.3)$ & $4.8(1.9-11.3)$ & $4.7(3.0-11.0)$ \\
\hline 2 & $14.4(8.3-20.9)$ & $14.5(9.0-22.6)$ & $10.1(3.2-14.7)$ & $9.0(3.9-12.3)$ \\
\hline 3 & $20.2(20.2-20.2)$ & $21.8(21.8-21.8)$ & - & - \\
\hline
\end{tabular}

Values are displayed as averages with corresponding minimum to maximum

$p<0.05)$. Distribution of MFC in respect to visual grading of fatty degeneration is displayed in Table 3 and Fig. 6.

\section{Discussion}

Our results show that $2 \mathrm{pt}-\mathrm{MRI}_{\mathrm{DIXON}}$ allows accurate quantification of MFC in a phantom study, as well as in patients and healthy volunteers.

Fat fractions derived from 2 pt-MRI $\mathrm{DIXON}_{\text {DIXON }}\left(\mathrm{FF}_{\mathrm{DIXON}}\right)$ and MRS ( FF $_{\text {MRS }}$ ) were significantly higher in the gastrocnemius muscle of patients with achillodynia compared with healthy volunteers. In contrast, visual assessment of fatty degeneration showed no significant difference between both groups. MFC was also significantly higher in study subjects with structural

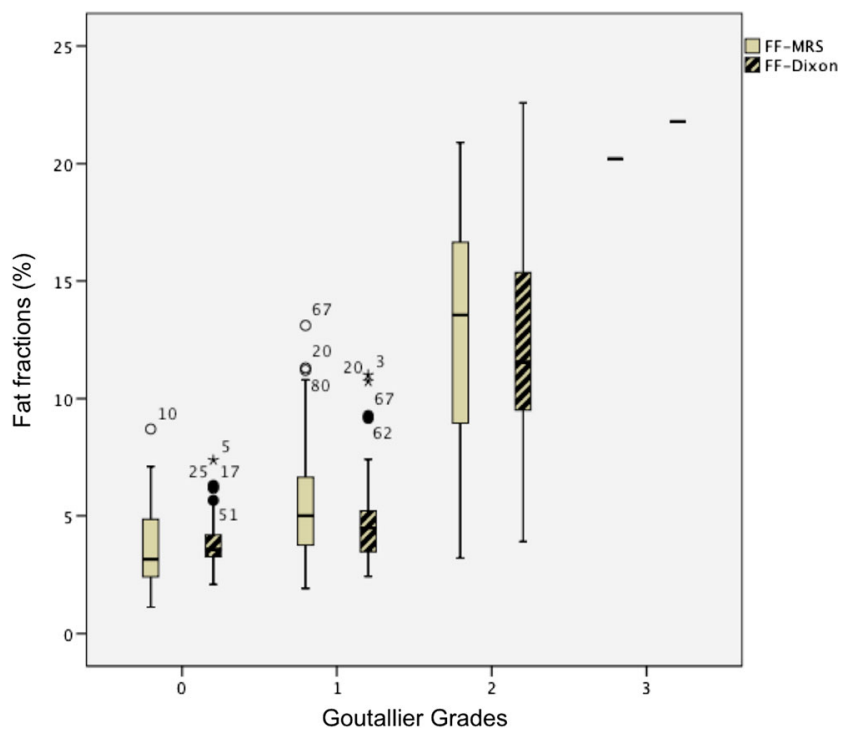

Fig. 6 Distribution of muscle fat content (MFC) as determined by 2point Dixon gradient-echo MRI ( $\left.\mathrm{FF}_{\mathrm{DIXON}}\right)$ and $\mathrm{MRS}\left(\mathrm{FF}_{\mathrm{MRS}}\right)$ along the $y$-axis in respect to visual grading of fatty degeneration according to Goutallier (grade 0-4) along the $x$-axis. None of the study subjects showed grade 4 fatty degeneration of muscle tissue. Data are displayed as box-and-whisker plots. Boundary of boxes closest to zero 25 th percentile, line within box median, boundary of boxes farthest from zero 75th percentile; error bars smallest and largest values within 1.5 box lengths of 25th and 75th percentiles damage of the Achilles tendon ( $>$ grade 1), compared with study subjects with an intact tendon.

Several studies have suggested that assessment of MFC may serve as a biomarker for detection of muscular insufficiency and prediction of post-surgical outcome in degenerative musculoskeletal disease $[2,7,9,10,16,18]$.

Recently, 2pt-MRI DIXON $_{\text {with water/fat separation has been }}$ proposed for fat quantification in patients with lower back pain [2], as well as in neuromuscular and metabolic disease [14, 19-21]. Advantages over MRS include a fast and volumetric data acquisition with both visualisation of anatomical structures and quantification of MFC in an ROI [22]. Howev-

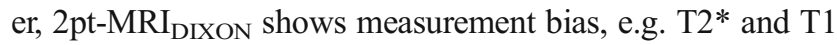
bias [23], which may degrade accuracy of fat quantification. We therefore evaluated the accuracy of a 2 pt-MRI $I_{\text {DIXON }}$ product sequence for quantification of MFC both in a controlled ex vivo setting and in patients and volunteers. Comparable to previous studies, the muscle/fat phantom provided a standard curve to validate the ex vivo measurements [23], whereas MRS served as the standard of reference for in vivo measurements of the MFC [24].

Our ex vivo results show that $\mathrm{FF}_{\text {DIXON }}$ correlated linearly and highly with the actual fat content in the phantom in a range from 0 to $100 \% \mathrm{MFC}$ (Fig. 3), which is consistent with previous studies evaluating $2 \mathrm{pt}-\mathrm{MRI}_{\mathrm{DIXON}}$ using fat/water phantoms [25, 26]. However, comparable to another study using fat/liver phantoms there was an overestimation of very small fat contents (0-4\%), which might be caused by the magnitude calculation of water-/fat-only images (noise bias) [3] affecting fat estimates according to the previously described algorithm (Eq. 1). Accordingly the correlation between $\mathrm{FF}_{\text {DIXON }}$ and the titrated fat content was worse within a clinical range of MCF from 0 to $30 \%$ (slope, 0.84).

Our in vivo results show that $\mathrm{FF}_{\text {DIXON }}$ closely and significantly matched $\mathrm{FF}_{\mathrm{MRS}}$, which is consistent with results from a recent study comparing different Dixon-based fat quantification methods using MRS as the standard of reference (Table 2). In contrast to the latter study, T1 bias of the 2ptDixon $_{\mathrm{GRE}}$ sequence was additionally addressed in our study by lowering the flip-angle to $5^{\circ}$ (proton-density weighting), 
resulting in a significant reduction of the mean measurement bias from $+1.6 \%$ to $+0.4 \%$ [27]. However, our in vivo results show - similar to the ex vivo study - a measurement bias of 2pt-MRI Dixon $_{\text {for very small and high }} \mathrm{FF}_{\text {DIXON }}$, probably due to noise bias [3] and single-peak modelling of fat $[28,29]$ respectively, resulting in a slope of 0.85 using linear regression analysis. Interestingly, MRS revealed a small interindividual range of $\mathrm{T} 2$ values in our study, which might explain the little importance of a $\mathrm{T} 2 *$ correction in estimations of MFC ( $\mathrm{FF}_{\mathrm{DIXON}}$ are not corrected for $\mathrm{T}^{*}$-bias), e.g. compared with liver-fat content. Moreover, noise bias of in vivo $\mathrm{FF}_{\text {DIXON }}$ was less prominent compared with the phantom study, which might be explained by signal contribution to in vivo fat-only images from surrounding subcutaneous and intramuscular fat.

The range of fat fractions described in the literature is highly variable, depending among others on factors like fat quantification technique, muscle type and disease. We demonstrate a range of MFC from $2.1 \%$ to $21.8 \%$ and from $3.0 \%$ to $11.0 \%$ for the gastrocnemius and tibialis anterior muscle respectively (Fig. 4), which is consistent with other studies using two-/multi-point Dixon techniques for quantification of MFC [2, 14, 19-21, 30]. Moreover, mean MFC values of our study were highly matching MFC values of a recent study evaluating MFC of calf muscles in respect to visual grading of fatty degeneration according to Goutallier (Fig. 6).

Our results show that $\mathrm{FF}_{\mathrm{DIXON}}$ and $\mathrm{FF}_{\mathrm{MRS}}$ were significantly higher in the gastrocnemius muscle of patients with achillodynia compared with healthy volunteers (Fig. 4). In contrast, visual assessment showed no significant difference between both groups, which is consistent with another study comparing quantitative with visual assessment of MFC in patients with lower back pain [31]. In our study, most study subjects showed no or only a subtle fatty muscle infiltration and significant overlap of quantitative MFC estimates was identified between Goutallier grade 0 and grade 1 . Consistent with a recent study [19], inter-observer agreement of visual assessment of fatty degeneration was good for all calf muscles. Our results show that agreement depends on MFC and were lower in the tibialis anterior muscle (mean MFC, 3.6 \pm 0.6) compared with the gastrocnemius muscle (mean MFC, $6.0 \pm 3.5$ ). This indicates that visual assessment hampers detection of subtle fatty muscle infiltration, whereas MFC derived from 2 pt-MRI DIXON $_{\text {might serve as a biomarker for early }}$ stages of muscle disease.

In our study (Fig. 5), MFC was significantly higher in study subjects with structural damage of the Achilles tendon (grade $>1$ ), compared with study subjects with intact Achilles tendon (grade $\leq 1$ ), which is consistent to a previously mentioned study performing a qualitative assessment of MFC and Achilles tendon quality [7]. However, we expand the results of the latter study by demonstrating a poor correlation between age and both the condition of the Achilles tendon and fatty muscle degeneration. Moreover, compared with the gastrocnemius muscle, no differences were identified between patients and healthy volunteers regarding MFC estimates of the tibialis anterior muscle, indicating that tendon integrity is an important factor of fatty muscle degeneration, more important than age.

Several limitations of our study have to be addressed. We performed Dixon-based fat quantification using a protonweighted two-point Dixon sequence but not a recently introduced multi-point Dixon MRI sequence with correction of chemical-shift inherited measurement bias, including T2* decay, multi-peak fat spectrum modelling and eddy currents [6]. However, we could not find those 2pt-MRI $\mathrm{Dixon}_{\text {rix }}$ related errors to significantly derogate the MFC estimates in both the ex vivo and in vivo analysis, which is consistent with a previous in vivo study evaluating both two-point and multipoint Dixon MRI for quantification of MFC [2]. On the other hand, the study cohort demonstrates only a small range of MFC (5.2\% $\% 3.5 \%)$ and the MRS technique used in this study did not consider an unsaturated lipids peak $>3.6 \mathrm{ppm}$, resulting in an underestimation of MRS based fat fractions similar to $\mathrm{FF}_{\text {DIXON }}$. The use of multi-point Dixon sequences addressing these confounding factors might therefore further increase the accuracy for muscle fat quantification once integrated into clinical routine. Moreover, the patient cohort has a wide age range. Whereas the control group (healthy volunteers) was matched according to sex, we were not able to accurately match the age of both groups. However, correlation of MFC with gender and age of study subjects revealed no significant or only a poor correlation indicating that the difference in age of both groups are of minor concern for the accuracy of our findings.

In summary, 2pt-MRI ${ }_{\text {DIXON }}$ allows for accurate quantification of MFC, outperforming visual assessment of calf muscle fat. Structural damage of the Achilles tendon is associated with a significantly higher MFC.

Acknowledgements The scientific guarantor of this publication is Professor Christian W. A. Pfirrmann. The authors of this manuscript declare no relationships with any companies, whose products or services may be related to the subject matter of the article. The authors state that this work has not received any funding. One of the authors has significant statistical expertise. No complex statistical methods were necessary for this paper. Institutional Review Board approval was obtained. Written informed consent was obtained from all subjects (patients) in this study. None of the study subjects or cohorts have been previously reported. Methodology: prospective, case-control study, performed at one institution.

\section{References}

1. Theodorou DJ, Theodorou SJ, Kakitsubata Y (2012) Skeletal muscle disease: patterns of MRI appearances. Br J Radiol 85:e1298-e1308

2. Fischer MA, Nanz D, Shimakawa A et al (2013) Quantification of muscle fat in patients with low back pain: comparison of multi-echo 
MR imaging with single-voxel MR spectroscopy. Radiology 266: $555-563$

3. Ma J (2008) Dixon techniques for water and fat imaging. J Magn Reson Imaging 28:543-558

4. Fischer MA, Nanz D, Reiner CS et al (2010) Diagnostic performance and accuracy of 3-D spoiled gradient-dual-echo MRI with water- and fat-signal separation in liver-fat quantification: comparison to liver biopsy. Investig Radiol 45:465-470

5. Schweitzer ME, Karasick D (2000) MR imaging of disorders of the Achilles tendon. AJR Am J Roentgenol 175:613-625

6. Sharma P, Maffulli N (2005) Tendon injury and tendinopathy: healing and repair. J Bone Joint Surg Am 87:187-202

7. Hoffmann A, Mamisch N, Buck FM et al (2011) Oedema and fatty degeneration of the soleus and gastrocnemius muscles on MR images in patients with Achilles tendon abnormalities. Eur Radiol 21:19962003

8. Munteanu SE, Barton CJ (2011) Lower limb biomechanics during running in individuals with achilles tendinopathy: a systematic review. J Foot Ankle Res 4:15

9. Bartolozzi A, Andreychik D, Ahmad S (1994) Determinants of outcome in the treatment of rotator cuff disease. Clin Orthop Relat Res 308:90-97

10. Gerber C, Schneeberger AG, Hoppeler H et al (2007) Correlation of atrophy and fatty infiltration on strength and integrity of rotator cuff repairs: a study in thirteen patients. J Shoulder Elbow Surg 16:691696

11. Raptis DA, Fischer MA, Graf R et al (2012) MRI: the new reference standard in quantifying hepatic steatosis? Gut 61:117-127

12. Dickson LC, Costain R, McKenzie D et al (2009) Quantitative screening of stilbenes and zeranol and its related residues and natural precursors in veal liver by gas chromatography-mass spectrometry. $\mathrm{J}$ Agric Food Chem 57:6536-6542

13. Dixon WT (1984) Simple proton spectroscopic imaging. Radiology 153:189-194

14. Fischmann A, Kaspar S, Reinhardt J et al (2012) Exercise might bias skeletal-muscle fat fraction calculation from Dixon images. Neuromuscul Disord 22:S107-S110

15. Pineda N, Sharma P, Xu Q et al (2009) Measurement of hepatic lipid: high-speed T2-corrected multiecho acquisition at 1H MR spectroscopy - a rapid and accurate technique. Radiology 252:568-576

16. Goutallier D, Postel JM, Bernageau J et al (1994) Fatty muscle degeneration in cuff ruptures. Pre- and postoperative evaluation by CTscan. Clin Orthop Relat Res 304:78-83

17. Lin L, Torbeck LD (1998) Coefficient of accuracy and concordance correlation coefficient: new statistics for methods comparison. PDA J Pharm Sci Technol 52:55-59
18. Gladstone JN, Bishop JY, Lo IK et al (2007) Fatty infiltration and atrophy of the rotator cuff do not improve after rotator cuff repair and correlate with poor functional outcome. Am J Sports Med 35:719-728

19. Alizai H, Nardo L, Karampinos DC et al (2012) Comparison of clinical semi-quantitative assessment of muscle fat infiltration with quantitative assessment using chemical shift-based water/fat separation in MR studies of the calf of post-menopausal women. Eur Radiol 22:1592-1600

20. Fischmann A, Hafner P, Gloor M et al (2012) Quantitative MRI and loss of free ambulation in Duchenne muscular dystrophy. J Neurol 260:969-974

21. Karampinos DC, Baum T, Nardo L et al (2012) Characterization of the regional distribution of skeletal muscle adipose tissue in type 2 diabetes using chemical shift-based water/fat separation. J Magn Reson Imaging 35:899-907

22. Rofsky NM, Lee VS, Laub G et al (1999) Abdominal MR imaging with a volumetric interpolated breath-hold examination. Radiology 212:876-884

23. Cassidy FH, Yokoo T, Aganovic L et al (2009) Fatty liver disease: MR imaging techniques for the detection and quantification of liver steatosis. Radiographics 29:231-260

24. Reeder SB, Robson PM, Yu H et al (2009) Quantification of hepatic steatosis with MRI: the effects of accurate fat spectral modeling. J Magn Reson Imaging 29:1332-1339

25. Kovanlikaya A, Guclu C, Desai C et al (2005) Fat quantification using three-point dixon technique: in vitro validation. Acad Radiol 12:636-639

26. Bernard CP, Liney GP, Manton DJ et al (2008) Comparison of fat quantification methods: a phantom study at 3.0T. J Magn Reson Imaging 27:192-197

27. Karampinos DC, Yu H, Shimakawa A et al (2011) T-corrected fat quantification using chemical shift-based water/fat separation: application to skeletal muscle. Magn Reson Med 66:1312-1326

28. Yu H, Shimakawa A, McKenzie CA et al (2008) Multiecho water-fat separation and simultaneous R2* estimation with multifrequency fat spectrum modeling. Magn Reson Med 60:1122-1134

29. Bydder M, Yokoo T, Hamilton G et al (2008) Relaxation effects in the quantification of fat using gradient echo imaging. Magn Reson Imaging 26:347-359

30. Gloor M, Fasler S, Fischmann A et al (2011) Quantification of fat infiltration in oculopharyngeal muscular dystrophy: comparison of three MR imaging methods. J Magn Reson Imaging 33:203-210

31. Mengiardi B, Schmid MR, Boos N et al (2006) Fat content of lumbar paraspinal muscles in patients with chronic low back pain and in asymptomatic volunteers: quantification with MR spectroscopy. Radiology 240:786-792 